Supporting Information for:

\title{
Folic Acid-Conjugated Radioluminescent Calcium Tungstate Nanoparticles as Radio-Sensitizers for Cancer Radiotherapy
}

Vincenzo J. Pizzuti†, Currie ${ }^{\ddagger}, \|$, Scott R. Clark ${ }^{\dagger}$, Anish P. Patel ${ }^{\dagger}$, Christopher R. Schorr ${ }^{\dagger}$, Yava Jones-Hall", Michael O. Childress $^{\Delta}$, Jeannie M. Plantenga ${ }^{\Delta}$, Nicholas J. Rancilio\#, Bennett D. Elzey, ${ }^{\ddagger}$, and You-Yeon Won ${ }^{*}, \dagger, \ddagger$

†Davidson School of Chemical Engineering, Purdue University, 480 W. Stadium Avenue, West Lafayette, Indiana 47907, United States

‡Purdue University Center for Cancer Research, Purdue University, 210 S. University Street, West Lafayette, Indiana 47907, United States

"Department of Comparative Pathobiology, Purdue University, 625 Harrison Street, West Lafayette, Indiana 47907, United States

$\triangle$ Department of Veterinary Clinical Sciences, Purdue University, 625 Harrison Street, West Lafayette, Indiana 47907, United States

\#Department of Veterinary Clinical Sciences, Auburn University, 1010 Wire Road, Auburn, Alabama 36849, United States

$\S$ These authors contributed equally to this work.

${ }^{*}$ Corresponding author. Email: yywon@ecn.purdue.edu

Number of Pages $=20$

Number of Schemes \& Figures $=12$

Number of Tables $=2$ (embedded in figures) 


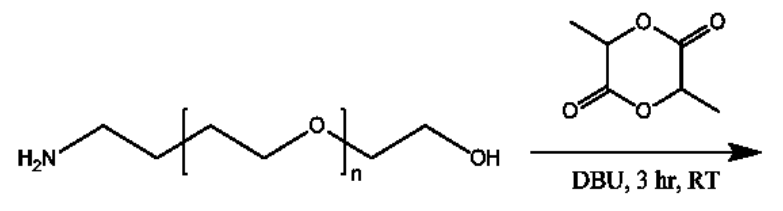<smiles>CC(C)OC(C)C(=O)C(C)OCCC(C)(C)OCCC(C)(C)CCN</smiles>

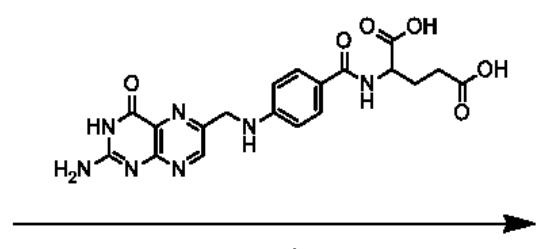

DCC, NHS, 24 hr, RT

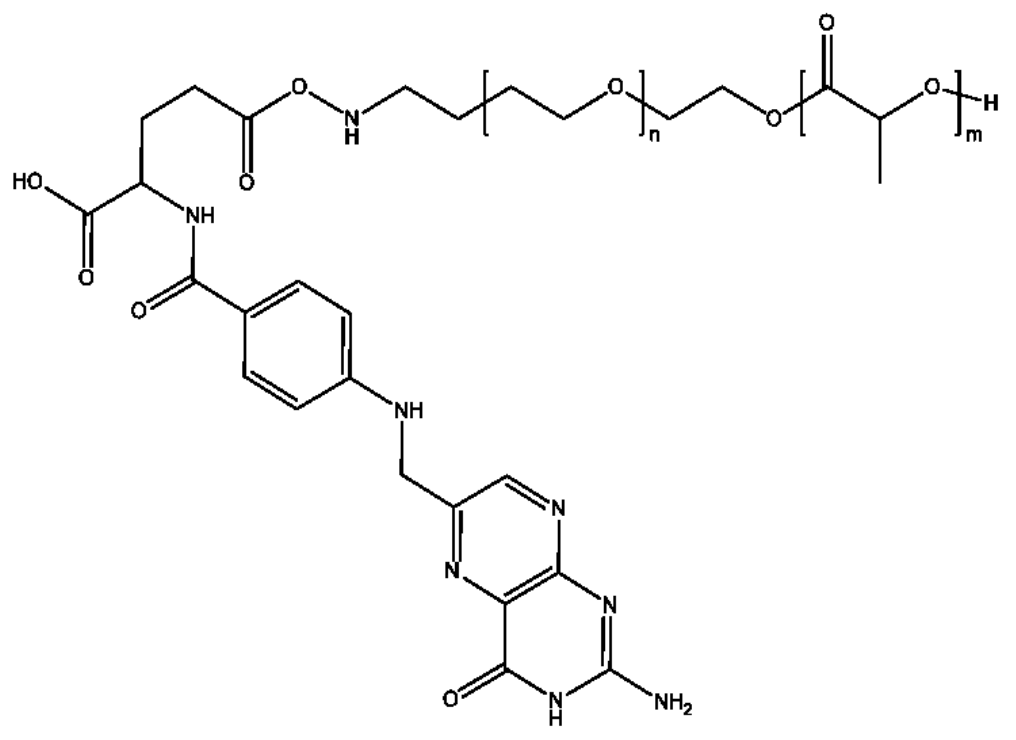

Scheme S1. Synthetic Pathw ay for FOL-PEG-PLA from $\mathrm{NH}_{2}-\mathrm{PEG}-\mathrm{OH}$. This novel pathw ay eliminated one reaction and one purification step from the original protocol ${ }^{1}$, allowing for easier synthesis and reduced cost and losses. 


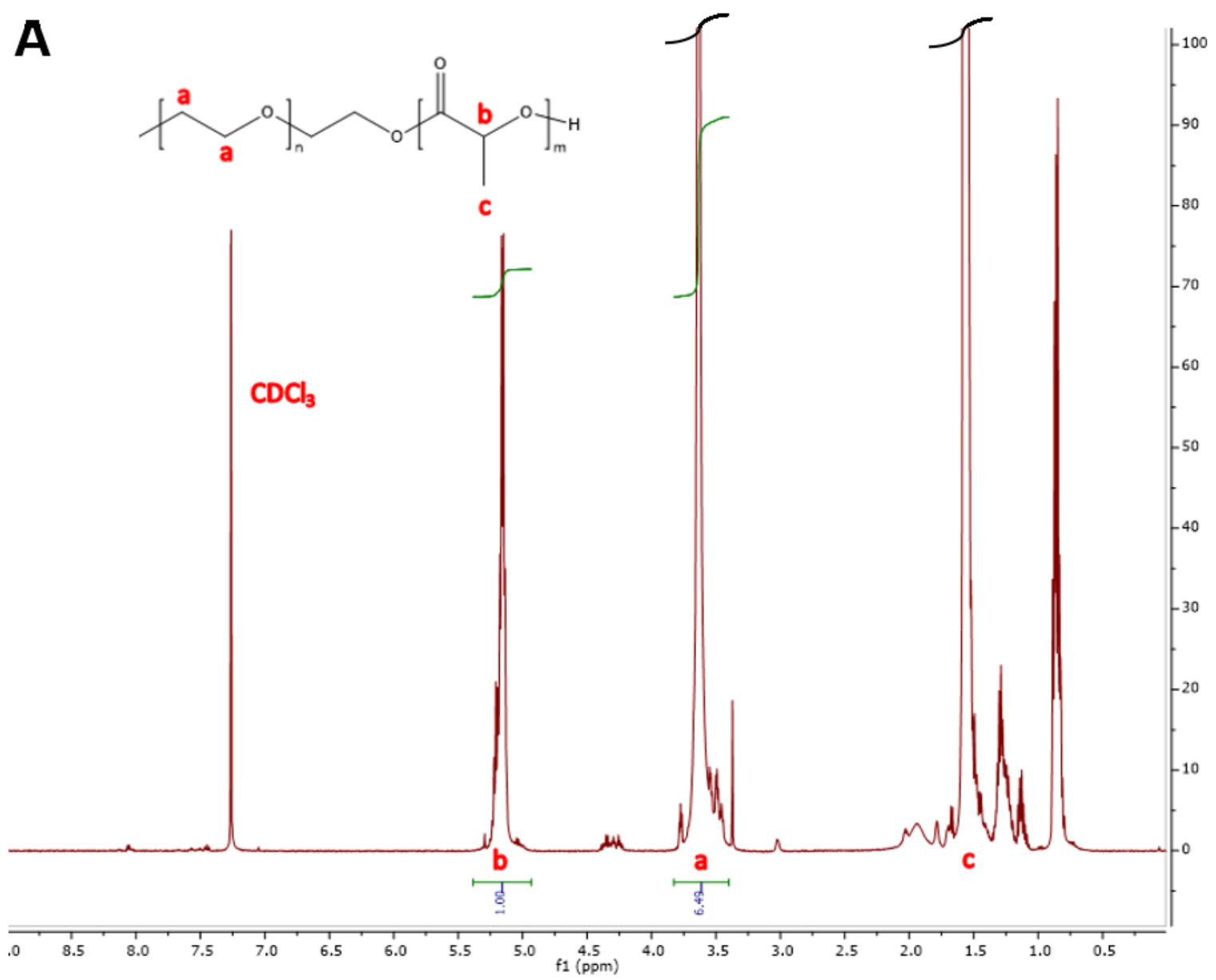




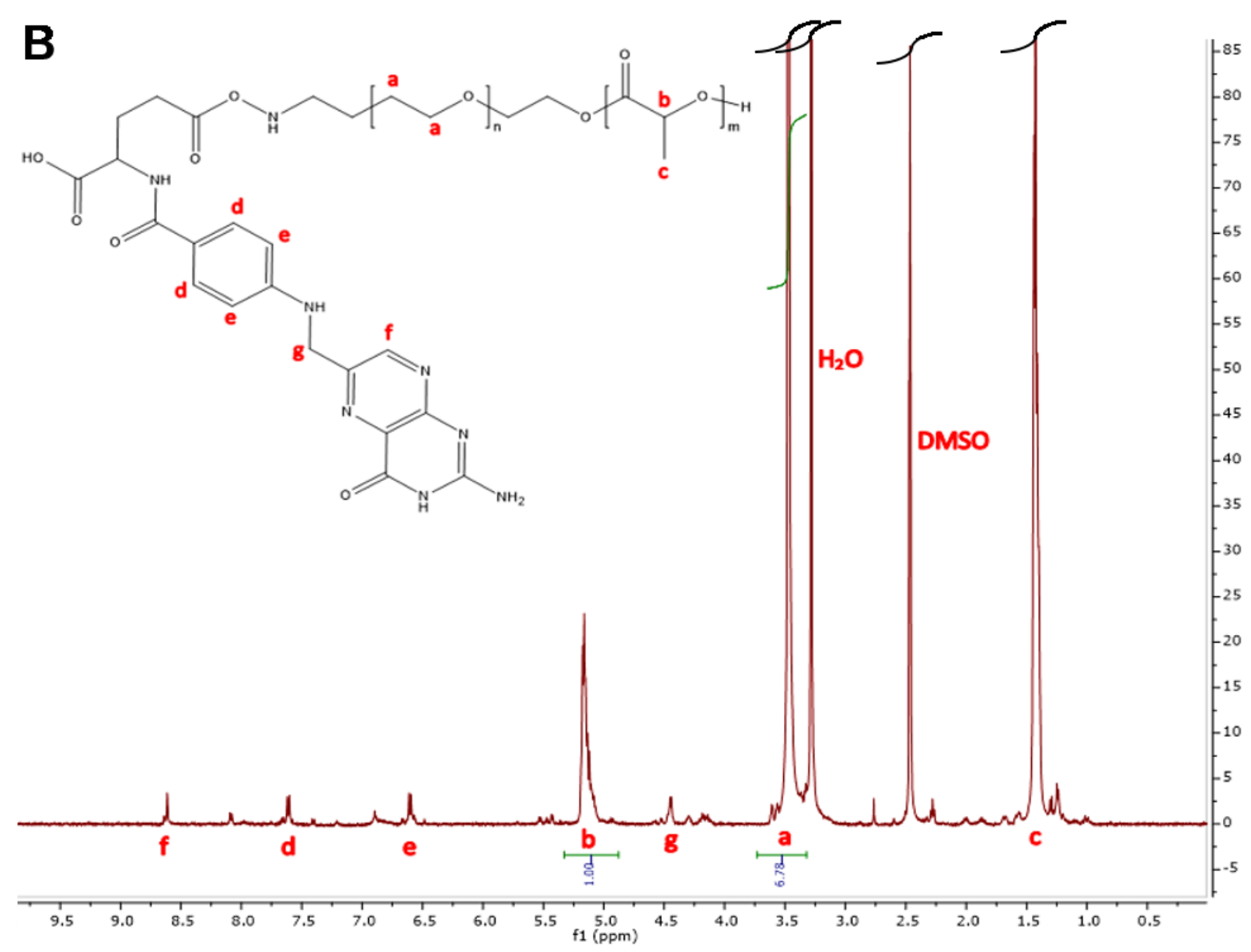

Figure S1. ${ }^{1} \mathrm{H}-\mathrm{NMR}$ Spectrum of Synthesized Polymers. (A) Structure of PEG-PLA was confirmed via proton NMR, and the $M_{n}$ of the polymerized PLA chain was estimated from integration to be 5,040 Da. (B) Structure of FOL-PEGPLA was confirmed via proton NMR, and the $\mathrm{Mn}_{\mathrm{n}}$ of the polymerized PLA chain was estimated from integration to be $4,850 \mathrm{Da}$. GPC results (not displayed) found that the polydispersity of PEG-PLA was 1.14 and FOL-PEG-PLA was 1.21. 

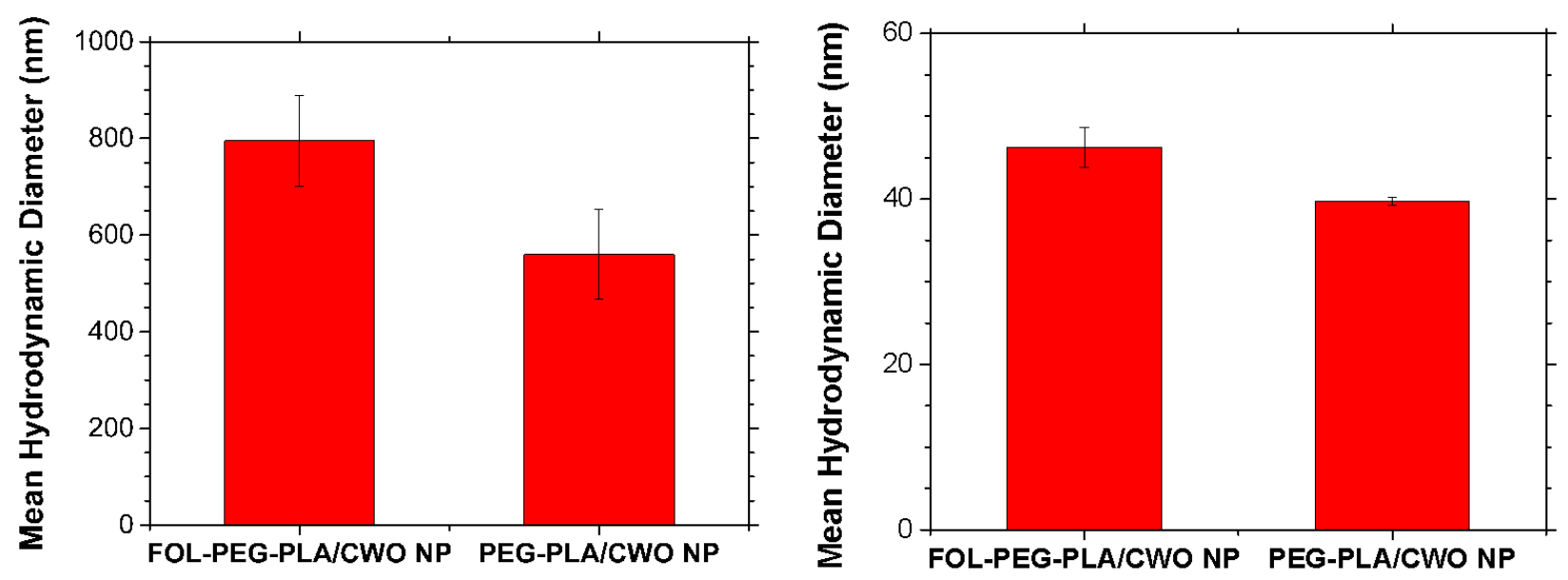

\begin{tabular}{|r|l|l|}
\hline & Unfiltered & Filtered \\
\hline PEG- & & \\
PLA/CWO & & \\
NP & $0.416 \pm 0.014$ & $0.078 \pm 0.007$ \\
\hline $\begin{array}{r}\text { FOL-PEG- } \\
\text { PLA/CWO }\end{array}$ & & \\
NP & $0.410 \pm 0.007$ & $0.172 \pm 0.030$ \\
\hline
\end{tabular}

Figure S2. DLS Measurements of PEG-PLANCWO NPS and FOL-PEG-PLAVCWO NPs. (Top Left) Unfiltered nanoparticle DLS results. (Top Right) Filtered nanoparticle DLS results. Mean effective diameters for PEG-PLA/CWO NPs and FOL-PEG-PLA/CWO NPs, produced as described in the Materials \& Methods. Error bars represent standard deviation. Unfiltered samples were left as prepared, and filtered samples were passed through a $200 \mathrm{~nm}$ PVDF syringe filter before analysis by DLS. For DLS runs, unfiltered particles were diluted ten-fold in PBS, and filtered particles w ere analyzed immediately after filtration without dilution. Table summarizes polydispersity values reported by DLS analysis (w ith standard deviations). $\mathrm{N}=3$. 

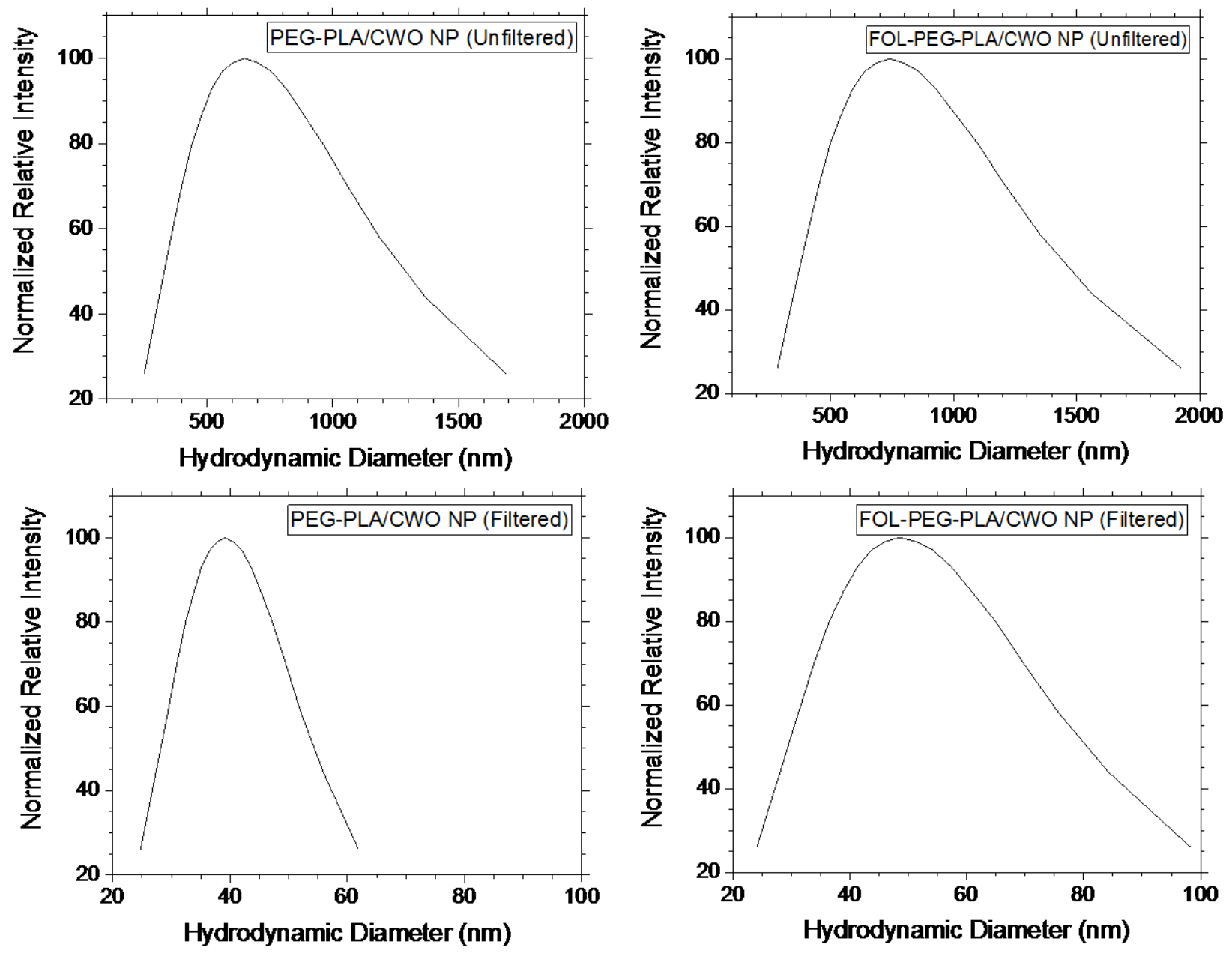

Figure S3. Representative Lognormal DLS Size Distributions of PEG-PLAVCWO NPs and FOL-PEG-PLAVCWO NPS. (Top Left) Unfiltered PEG-PLA/CWO NP DLS lognormal particle size distribution. (Top Right) Unfiltered FOL-PEGPLA/CWO NP DLS lognormal particle size distribution. (Bottom Left) Filtered PEG-PLA/CWO NP DLS lognormal particle size distribution. (Bottom Right) Filtered FOL-PEG-PLA/CWO NP DLS lognormal particle size distribution. Filtered samples were passed through a $200 \mathrm{~nm}$ PVDF syringe filter before analysis by DLS. Unfiltered particles were diluted ten-fold in PBS, and filtered particles were analyzed immediately after filtration without dilution. Each plot is a representative example taken from one of the $\mathrm{N}=3$ samples displayed in Figure $\mathrm{S} 2$. 


\section{Supplemental Material for Mechanism of Action Examination}

Hypothesis (1) from the main text was tested by exposing HN31 cells to FOL-PEG-PLA/CWO NPs with varying folate densities, PEG-PLA/CWO NPs, free folic acid, and negative and positive controls (PBS and 5-aminolevulinic acid, respectively) for 4 hours and subsequently measuring intracellular and extracellular PPIX levels. PPIX was chosen as a target molecule because, as mentioned in the main text, it is an endogenous photosensitizer with the ability to produce ROS upon excitation from a UV/blue light source. Although folic acid is not known to induce photosensitization of cancer cells, the haem/iron metabolic pathways do interact with the folic acid pathway via two proteins: SLC46A1 and ferritin². Folic acid competes with exogenous iron sources for transcellular transport via SLC46A1 ${ }^{3}$. High levels of ferritin upregulate folate-specific enzymes ${ }^{4-5}$, so the converse relationship with high folic acid levels could potentially increase ferritin levels, leading to reduced free iron and decreased conversion of PPIX to haem. Examination to determine if folic acid functionalization would induce changes in PPIX levels in the cells was warranted since PPIX is the immediate metabolic precursor to haem ${ }^{6-7}$.

The resultant PPIX levels are displayed in Figure S4. Only the positive control (5-ALA) showed significant enhancement in PPIX levels compared to the negative control. These data suggested that the increase in efficacy from FOL-PEG-PLA/CWO NPs compared to PEG-PLA/CWO NPs was not due to increases in PPIX levels initiated by FOL-PEG-PLA/CWO NPs. 

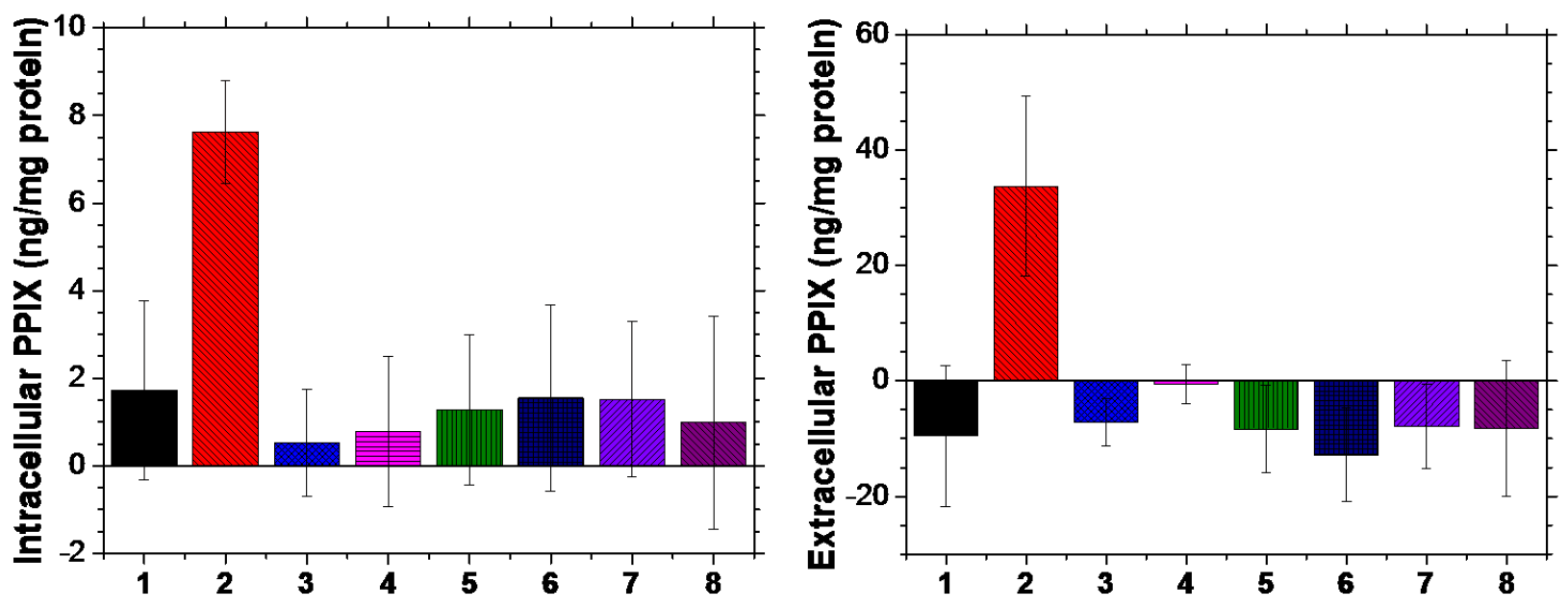

Figure S4. Intracellular PPIX Levels in Response to NP Exposure. Intracellular and extracellular PPIX levels in HN31 cells with exposure to (1) PBS (neg. control), (2) 5-ALA (pos. control,1 mM), (3) PEG-PLA/CWO NPs, (4) free folic acid (0.2 mM), (5) 10\% FOLPEG-PLA Micelles (empty micelles without CWO NPs and $10 \%$ of PEG chains by weight functional ized with folic acid), (6) 10\% FOLPEG-PLA/CWO NP (standard FOL-PEG-PLA/CWO NPs with $10 \%$ by weight of PEG chains functionalized with folic acid), (7) $25 \%$ FOL-PEG-PLA/CWO NP ( $25 \%$ by weight of PEG chainsfunctionalized with folic acid), and ( 8 ) $60 \%$ FOL-PEG-PLA/CWO NPs ( $60 \%$ by weight of PEG chains functionalized with folic acid) (all CWO samples were at a concentration of $0.2 \mathrm{mg} / \mathrm{mL}$ ) for 4 hours. Measurements were conducted using a fluorescence spectrophotometer $(405 \mathrm{~nm}$ ex. $/ 630 \mathrm{~nm} \mathrm{em}$.) on cell extracts (intracellular) or used cell culture media (extracellular) from $2.0 \times 10^{5}$ cells per well. Signals were normalized to total cellular protein measured via the BCA assay. Raw measurement values of fluorescence for extracellular samples were corrected for background fluorescence from materials present in the culture medium, and these measurements are displayed in supplemental Figure S 5 . All error bars represent standard deviations. *Only the positive control was significantly different compared to negative control in each case, indicated by $p<$ 0.05 compared with the negative control within each run using two-tailed Student's t-test $(\mathrm{N}=6)$. Note: Experiment conducted with unfiltered particles. 


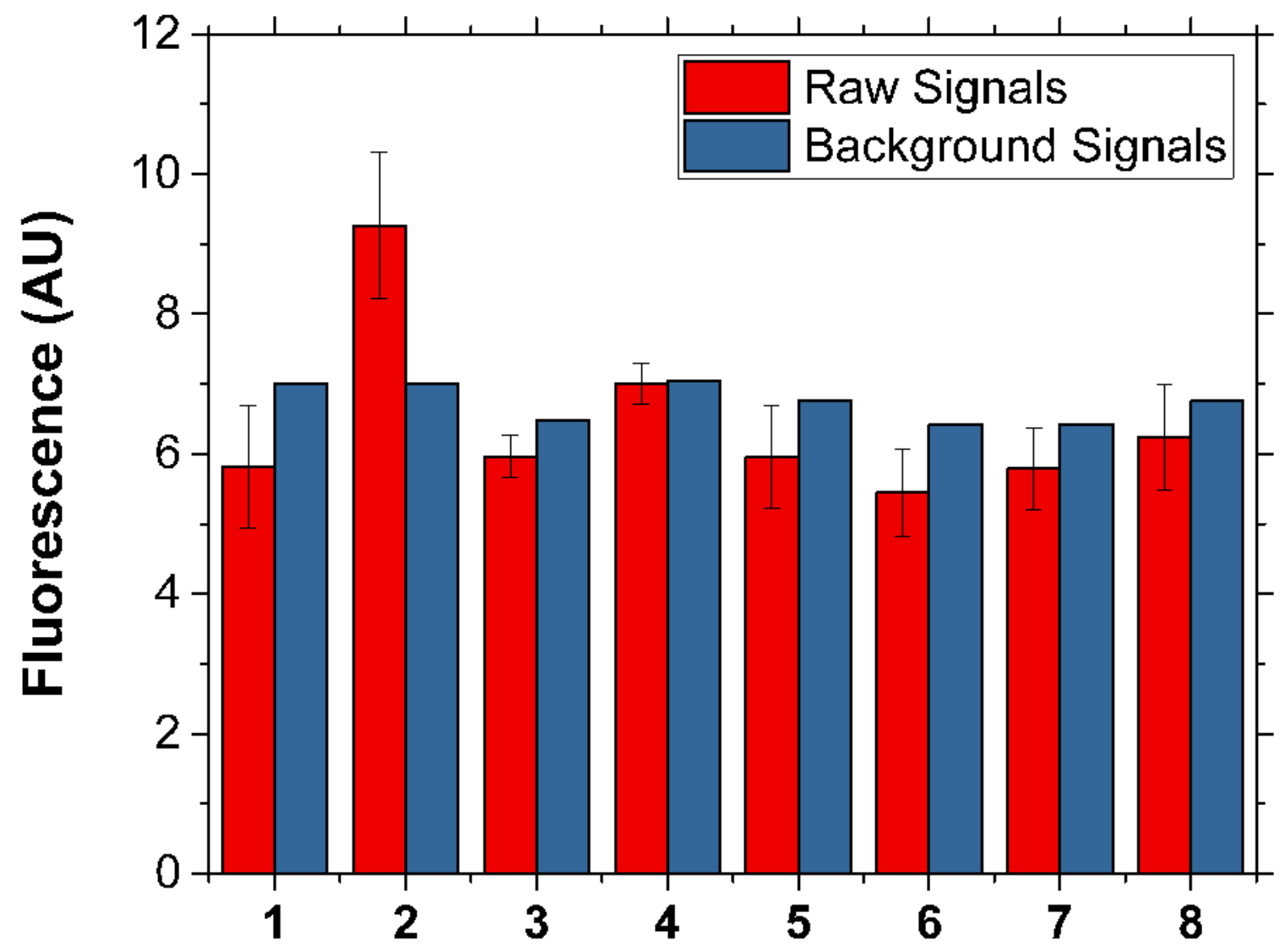

Figure S5. Raw PPIX Measurements for Extracellular PPIX Data. Displayed are the raw signals for the extracellular PPIX measurements alongside their background signals for fluorescence at $630 \mathrm{~nm}$ with $405 \mathrm{~nm}$ excitation. Group numbers are the same as in Figure S4. Error bars represent standard deviations. Note that the background signals are within error of the measurements for all except the positive control, which is why Fig. S4 displays extracellular PPIX levels that are slightly below zero. $\mathrm{N}=6$. 
Hypothesis (2) was tested using flow cytometry experiments that were conducted with HN31 cells exposed to FOL-PEG-PLA/CWO NPs and PEG-PLA/CWO NPs loaded with coencapsulated PPIX as a fluorescent marker. After a 4-hour incubation with the NPs, cells were analyzed for fluorescence at $630 \mathrm{~nm}$ (using $405 \mathrm{~nm}$ excitation). Figure $\mathrm{S} 6$ displays the histogram results of this experiment. The calculated uptake efficiencies were $84.6 \%$ and $81.3 \%$ for PEG-PLA/CWO NPs and FOL-PEG-PLA/CWO NPs, respectively. This indicates that PEGPLA/CWO NPs and FOL-PEG-PLA/CWO NPs are taken up equally well and suggests that the increases in efficacy in the FOL-PEG-PLA/CWO NP treatment group compared to the PEGPLA/CWO NP treated group is not due to increased cell uptake/internalization. 


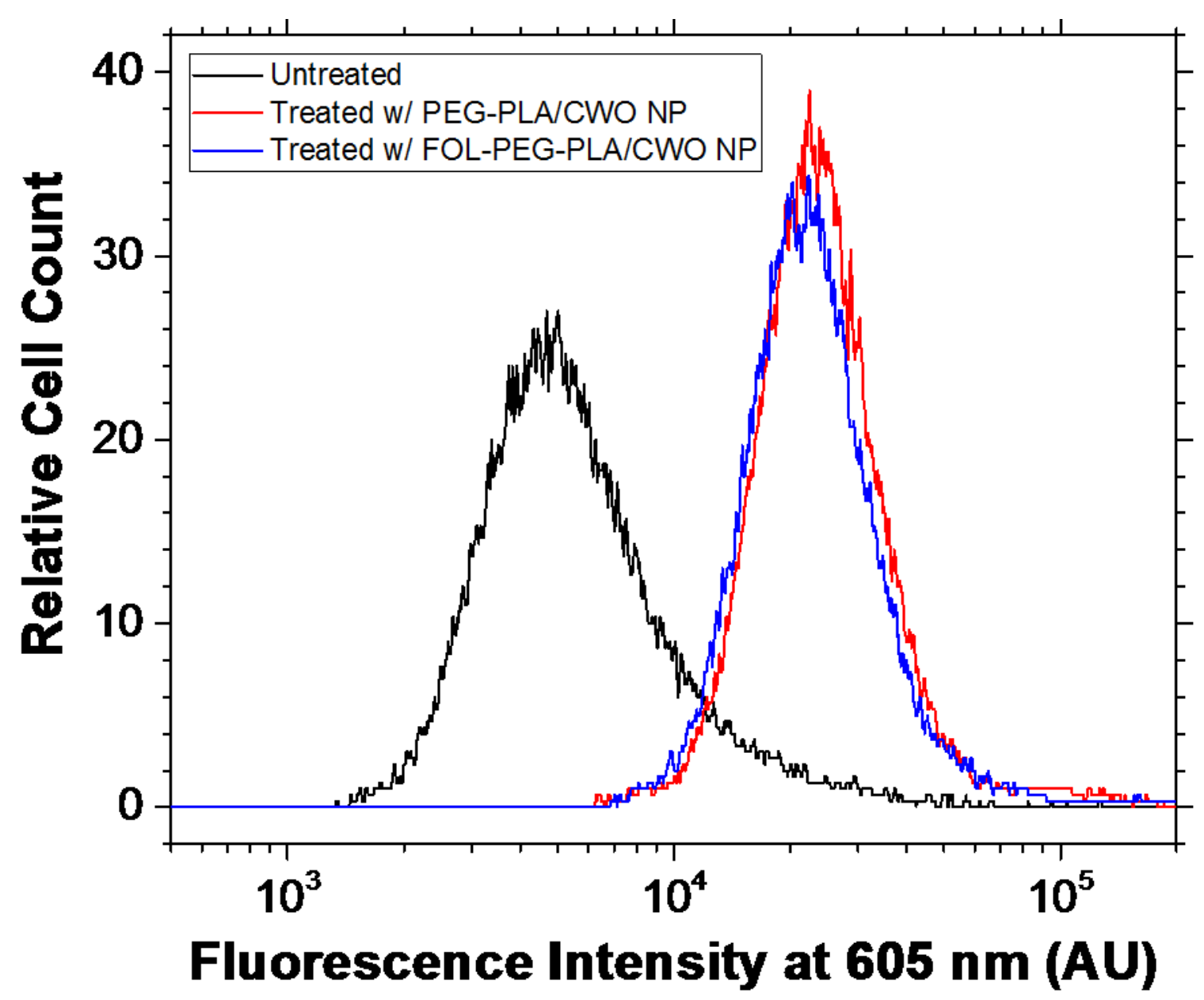

Figure S6. Uptake of Nanoparticles by HN31 Cells. HN31 Cellswere exposed to PEG-PLA/CWO NPsand FOL-PEG-PLA/CWO NPs with co-encapsulated PPIXasa fluorophore for uptake measurementat a CWO concentration of $0.2 \mathrm{mg} / \mathrm{mL}$. Cellswere incubated with NPsfor 4 hours, washed, and removed from the wells. Cellswere then analyzed using flow cytometry using standard techniques with $405 \mathrm{~nm}$ excitation and $630 \mathrm{~nm}$ emission measurements. The resultant histogram of cell count versusfluorescen ce intensity is displayed above. Overall uptake efficiencies were $84.6 \%$ and $81.3 \%$ for PEG-PLA/CWO NPsand FOL-PEG-PLA/CWO NPs, respectively. Note: Experiment conducted with filtered particles. 
To test hypothesis (3), a confocal imaging experiment was conducted in HN31 cells. Here, the cells were cultured directly on microscope cover slips until they reached around $70-80 \%$ confluence, at which point they were exposed to FOL-PEG-PLA/CWO NPs and PEG-PLA/CWO NPs loaded with co-encapsulated PPIX (as a fluorescent tracker of the NPs) for 4 hours. Afterward, the cells were washed 3 times with PBS and fixed using cold methanol. The cover slips were then mounted onto slides and imaged using DIC with a $488 \mathrm{~nm}$ laser and for fluorescence at $630 \mathrm{~nm}$ using $405 \mathrm{~nm}$ laser excitation. The images obtained are displayed in Figure S7. Inspection of the images reveals no immediate discernable difference between FOLPEG-PLA/CWO NP and PEG-PLA/CWO NP treated groups. FFT transformations were performed and analyzed for average intensities, and the groups were not significantly different (see Figure S8). Additionally, an average pixel intensity measurement for the cell nuclei in each group was conducted and found that there was no difference in the pixel intensities found within the nuclei of each cell (see Figure S9). From this analysis, it is reasonable to state that there was no difference observed in the intracellular trafficking of FOL-PEG-PLA/CWO NPs compared to PEG-PLA/CWO NPs, and therefore is likely not the reason for the increase in efficacy observed for FOL-PEG-PLA/CWO NPs. 

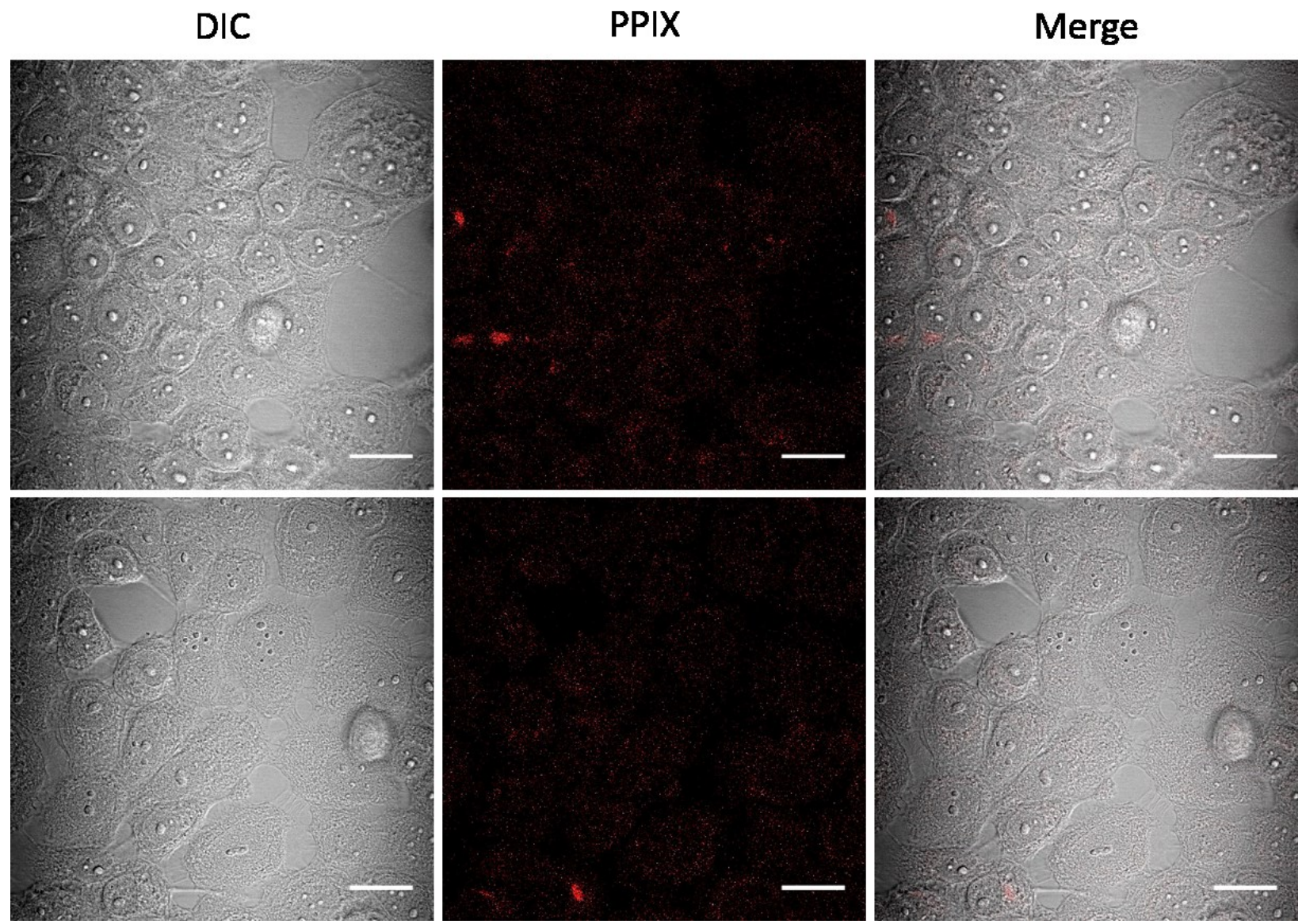

Figure S7. Confocal Images of Labeled PEG-PLA/CWO NPs and FOL-PEG-PLA/CWO NPs in HN31 Cells. (Top) HN31 cells exposed to PPIX-labeled FOL-PEG-PLA/CWO NPs. (Bottom) HN31 cells exposed to PPIX-labelled PEG-PLA/CWO NPs. Scale bars $=20 \mu \mathrm{m}$. Cellswere cultured directly on microscope coversl ips until reaching approximately $80 \%$ confluence. Cellswere then incubated with labelled nanoparticle solutions at a CWO concentration of $0.2 \mathrm{mg} / \mathrm{mL}$ for 4 hoursin the dark. Cellswere washed three times with PBS and fixed with cold methanol and then mounted onto microscope slidesand imaged using a Zeiss LSM 880 Upright Confocal at $63 x$ using an oil immersion lens. DIC images were taken using a $488 \mathrm{~nm}$ laser, and PPIX imageswere taken using a $405 \mathrm{~nm}$ laser with a $600-650 \mathrm{~nm}$ emission filter. For PPIX imagesfor both samples, contrast was enhanced by the same amount to improve visibility. Note: Filtered particleswere used for thisexperiment. 

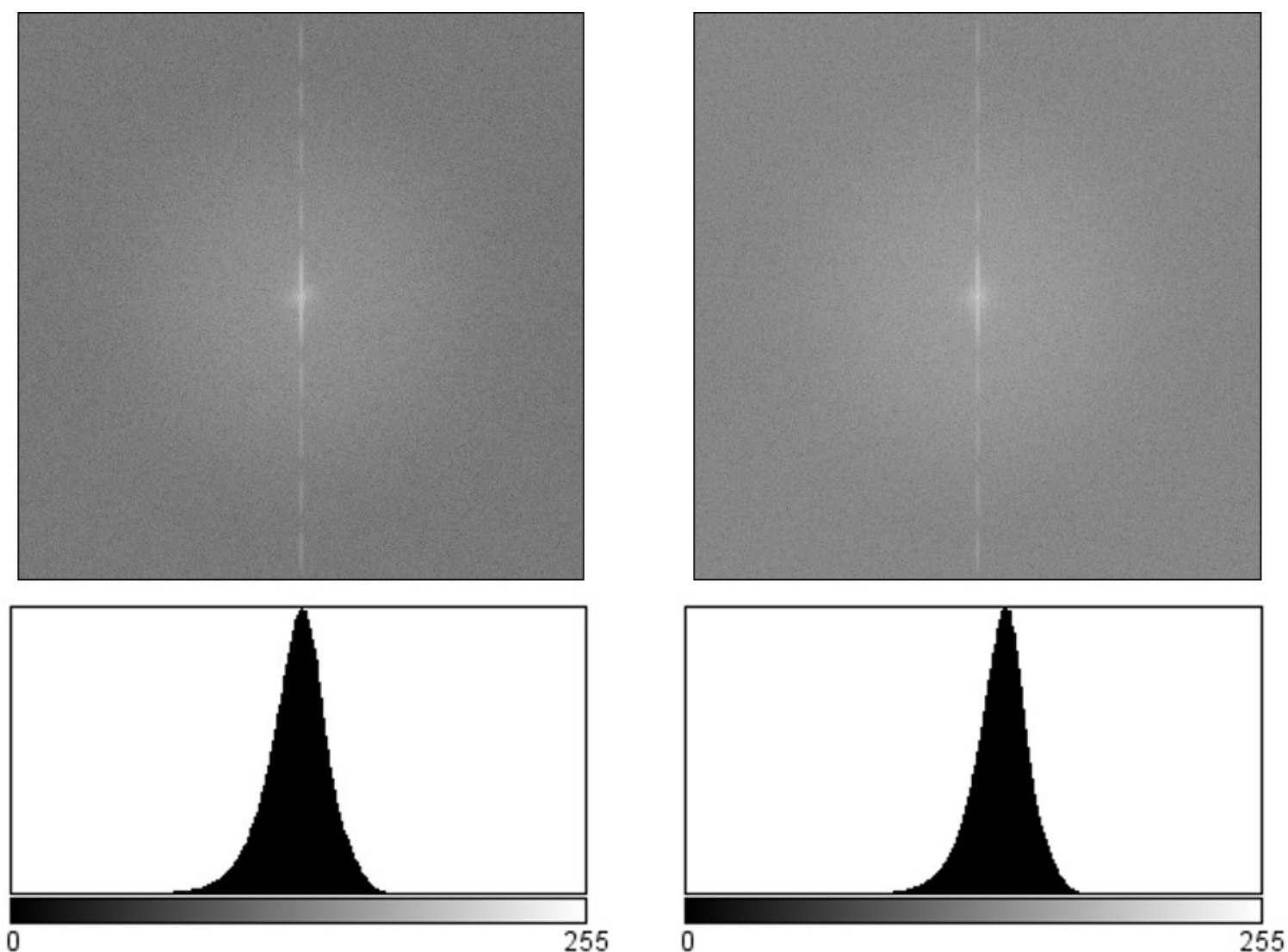

$\begin{array}{ll}\text { Count: } 1048576 & \text { Min: } 1 \\ \text { Mean: } 126.780 & \text { Max: } 254 \\ \text { StdDev: } 14.213 & \text { Mode: } 129 \text { (34898) }\end{array}$

Count: 1048576

Mean: 139.484

StdDev: 12.661
Min: 1

Max: 254

Mode: 141 (38908)

Figure S8. FFT of Confocal PPIX Channel Images from Figure S7. (Upper Left) FFT of PEG-PLA/CWO NP confocal PPIX channel image. (Upper Right) FFT of FOL-PEG-PLA/CWO NP confocal PPIX channel image. (Low er Left) Histogram results of FFT of PEG-PLA/CWO NP sample. (Low er Right) Histogram results of FFT of FOL-PEGPLA/CWO NP sample. ImageJ w as used to conduct FFT of each image. Then histograms w ere generated for each sample. No significant difference is apparent from the FFT histograms. 


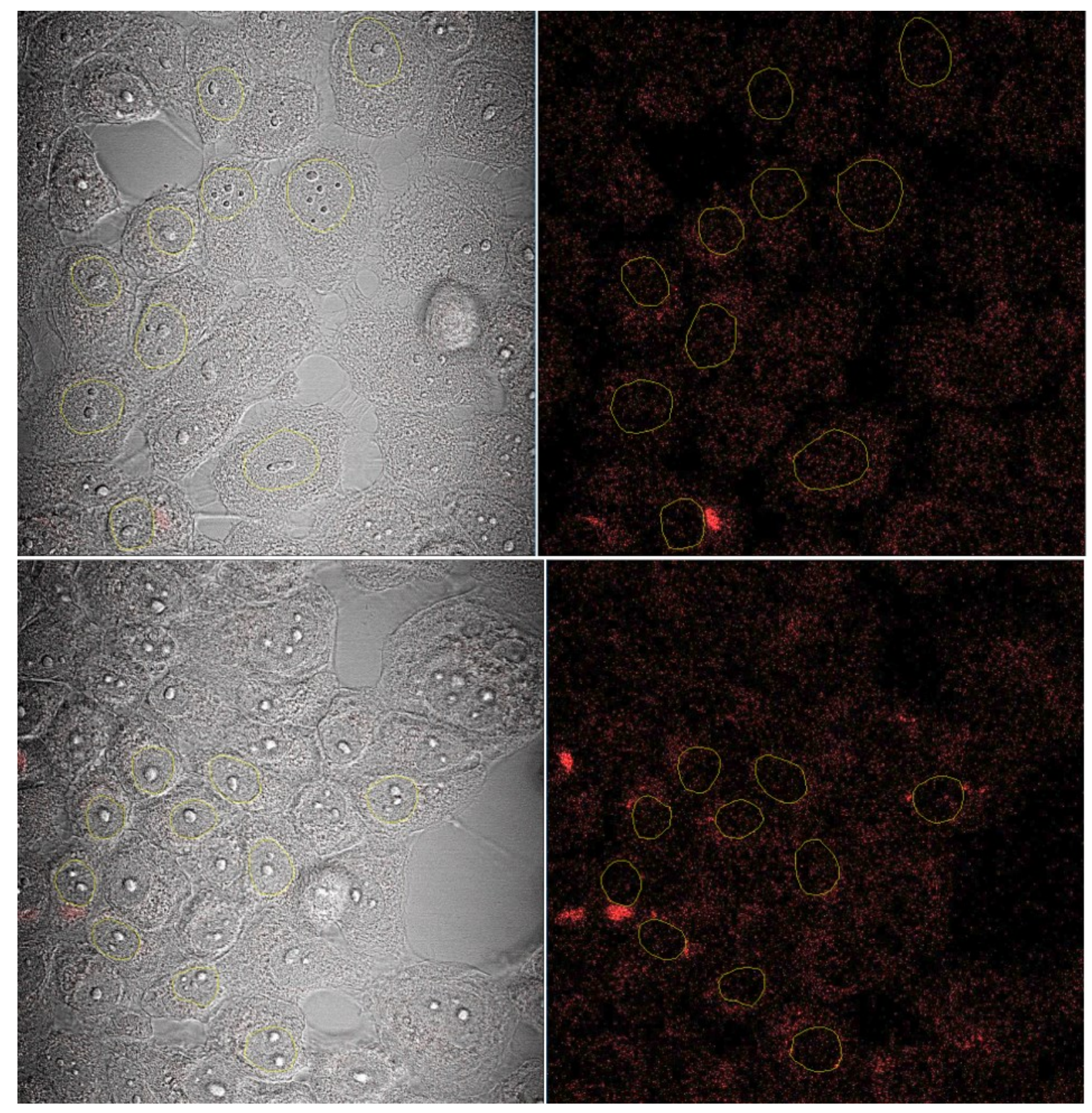

\begin{tabular}{|r|r|r|r|}
\hline & Area (pixels $^{2}$ ) & Mean (A.U.) & Std. Dev. (A.U.) \\
\hline PEG- & & & \\
PLA/CWO & & & \\
NP & 83099 & 11.822 & 22.304 \\
\hline $\begin{array}{r}\text { FOL-PEG- } \\
\text { PLA/CWO }\end{array}$ & & & \\
NP & 54803 & 12.796 & 21.722 \\
\hline
\end{tabular}

Figure S9. Intra-nuclear PPIX Intensities. (Top) PEG-PLAVCWO NP sample (Bottom) FOL-PEGPLA/CWO NP sample. In ImageJ, boundaries were manually drawn and mean pixel intensities were measured. The values are displayed in the table below the images above. Areas represent total area of all 10 nuclei analyzed for each sample and are measured in square pixels. The mean and standard 
deviation values are measured in arbitrary units of pixel intensity. No clear difference in intra-nuclear intensities were observed. 
Hypothesis (4) was examined by performing a classical radiobiological assay comparing sublethal damage repair (SLDR) in HN31 cells treated with FOL-PEG-PLA/CWO NPs, PEGPLA/CWO NPs, and PBS and subsequently irradiated with a split X-ray dose at various time intervals. The goal of this experiment was to determine if there was a substantial difference in SLDR kinetics or maximal repair for cells in each of the treatment groups, indicated by clonogenic survival of HN31 cells in each treatment category. The results are displayed in Figure S10. As seen in the figure, the curve for each of the treatment groups is shifted from the others, consistent with the initial clonogenic efficacy data found in Figure 5 in the main text. However, the shapes of the curves (SLDR kinetics) and increase in survival (SLD maximal repair) observed indicated that there is little or no difference in SLDR for the treatment groups. From this, we concluded that SLDR differences could not explain the difference in efficacy between FOL-PEG-PLA/CWO NP- and PEG-PLA/CWO NP-treated cells. 


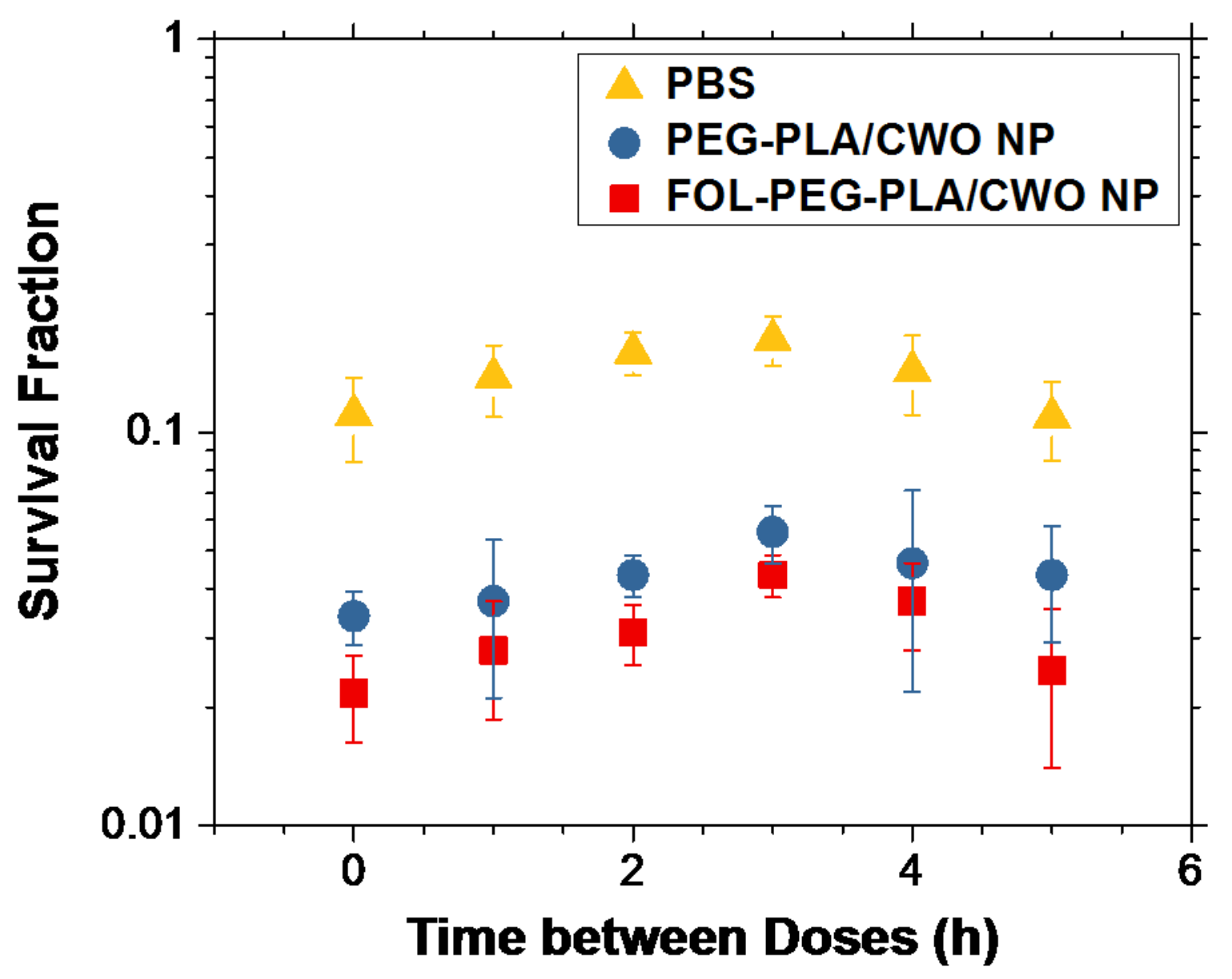

Figure S10. Sub-lethal Damage Repair Measured by Clonogenic HN31 Survival after Split Dose X-ray. HN31 cells were seeded in 6-well culture dishes at a density of $1.0 \times 10^{3}$ per well. After 4-hour incubation with PBS, PEG-PLA/CWO NPs or FOL-PEG-PLA/CWO NPs, cells were exposed to 2 doses of 2 Gy X-ray radiation separated by various times with a $320 \mathrm{keV}$ X-ray irradiator at approximately $2 \mathrm{~Gy} / \mathrm{min}$. Irradiated cells were cultured for 14 days. Colonies of more than 50 daughter cells in culture were counted $(\mathrm{N}=3$ wells per group). Error bars represent standard deviations. Note: Experiment conducted using unfiltered particles. 


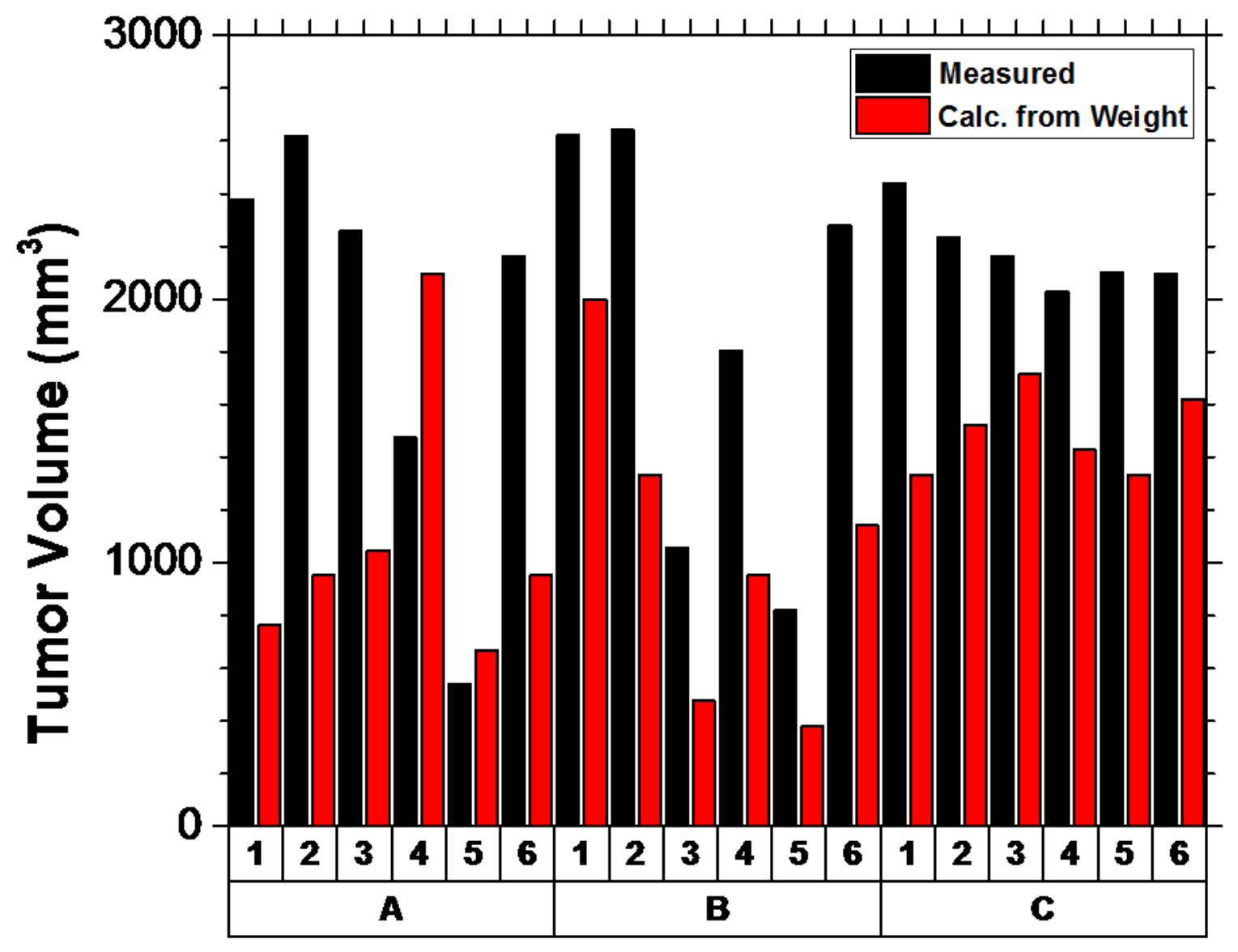

Figure S11. Measured Tumor Volume vs. Weight-calculated Volume in Mouse Study. In the mouse study reported, tumor volumes were measured with digital calipers for length $(L)$ and width $(W)$ in two dimensions. Mice were treated with (A) PBS + X-ray, (B) PEG-PLAVCWO NP + X-ray, and (C) FOL-PEG$\mathrm{PLA} / \mathrm{CWO} \mathrm{NP}+\mathrm{X}$-ray. Volume was calculated as $\mathrm{V}=\left(\mathrm{L} \times \mathrm{W}^{2}\right) \times \pi / 6$. For tumor volume estimations from tumor weight, a density of $1.05 \mathrm{~g} / \mathrm{cc}$ was used. Details of tumor volume calculations for the mouse study displayed in Figure 9 of the main text. The two-dimensional tumor measurements (with the assumption of ellipsoidal symmetry) overestimated the tumor volume considerably compared to weight-based estimates. The reason for this is the asymmetry of the tumors involved in these experiments. Some mice in the studies formed blisters on the tumor xenografts, which burst before euthanasia, and contributed to the discrepancy in tumor volume estimates due to the introduction of asymmetry caused by the apparent "larger" volume from the blister liquid. 


\section{Supporting Information References:}

1. Sung Duk Jo, J. L., Min Kyung Joo, Vincenzo J. Pizzuti, Nicholas J. Sherck, Slgi Choi, Beom Suk Lee, Sung Ho Yeom, Sang Yoon Kim, Sun Hwa Kim, Ick Chan Kwon, You-Yeon Won, PEG-PLA-Coated and Uncoated Radio-Luminescent CaWO4 Micro- and Nanoparticles for Concomitant Radiation and UVA/Radio-Enhancement Cancer Treatments. ACS Biomaterials Science \& Engineering 2018, 4 (4), 1445 1462.

2. Bailey, L. B.; Stover, P. J.; McNulty, H.; Fenech, M. F.; Gregory, J. F.; Mills, J. L.; Pfeiffer, C. M.; Fazili, Z.; Zhang, M.; Ueland, P. M.; Molloy, A. M.; Caudill, M. A.; Shane, B.; Berry, R. J.; Bailey, R. L.; Hausman, D. B.; Raghavan, R.; Raiten, D. J., Biomarkers of Nutrition for Development -Folate Review. The Journal of Nutrition 2015, 145 (7), 1636S-1680S. DOI: 10.3945/jn.114.206599.

3. Laftah, A. H.; Latunde-Dada, G. O.; Fakih, S.; Hider, R. C.; Simpson, R. J.; McKie, A. T., Haem and folate transport by proton-coupled folate transporter/haem carrier protein 1 (SLC46A1). British Journal of Nutrition 2008, 101 (8), 1150-1156. DOI: 10.1017/S0007114508066762.

4. Oppenheim, E. W.; Adelman, C.; Liu, X.; Stover, P. J., Heavy Chain Ferritin Enhances Serine Hydroxymethyltransferase Expression and de Novo Thymidine Biosynthesis. Journal of Biological Chemistry 2001, 276 (23), 19855-19861. DOI: 10.1074/jbc.M100039200.

5. Woeller, C. F.; Fox, J. T.; Perry, C.; Stover, P. J., A Ferritin-responsive Internal Ribosome Entry Site Regulates Folate Metabolism. Journal of Biological Chemistry 2007, 282 (41), 29927-29935. DOI: 10.1074/jbc.M706264200.

6. Berlin, N. I.; Neuberger, A.; Scott, J. J., The metabolism of $\delta$-aminolaevulic acid. 1 . Normal pathways, studied with the aid of $\langle$ sup $>15</$ sup $>$ N. Biochemical Journal 1956, 64 (1), 80-90. DOI: 10.1042/bj0640080.

7. Berlin, N. I.; Neuberger, A.; Scott, J. J., The metabolism of $\delta$-aminolaevulic acid. 2. Normal pathways, studied with the aid of $<$ sup $>14<$ /sup $>$ C. Biochemical Journal 1956, 64 (1), 90-100. DOI: 10.1042/bj0640090. 